\title{
Pattern matching: Effects of size ratio, angular difference in orientation, and familiarity
}

\author{
AXEL LARSEN \\ Copenhagen University, Copenhagen, Denmark
}

\begin{abstract}
Matching of simultaneously presented patterns with respect to shape regardless of differences in size and orientation was investigated using reaction time methods. When a new pair of unfamiliar random polygons was presented on each trial, matching reaction times in a number of experimental conditions showed strong linear and additive effects of the linear size ratio and the angular difference in orientation between the members of a stimulus pair. However, when the same few random polygons were used over an extended number of trials, the pattern of linear and additive effects degenerated. The results provide a basis for resolving some conflicts in the literature on mental transformations of size and orientation.
\end{abstract}

Matching a pair of visual patterns with respect to shape regardless of differences in size or orientation is a fundamental task in visual cognition. Introspection suggests that the task may be done by (1) encoding a visual representation of one of the patterns as a mental image, (2) transforming this image such that the represented size and orientation gradually change to those represented in the visual impression of the other pattern, and (3) matching this impression against the transformed mental image. Reaction time studies have supported this sort of account. Shepard and Metzler (1971) found the time taken to recognize that two perspective drawings depicted objects of the same three-dimensional shape to be an increasing linear function of the angular difference in orientation between the two objects. Bundesen and Larsen (1975) found the time taken to recognize that two random figures were the same in shape to be an increasing linear function of the ratio of their linear sizes. Moreover, using patterns differing in both size and orientation, Bundesen, Larsen, and Farrell (1981) found matching reaction times to be increasing linear functions of both linear size ratio and angular difference in orientation such that effects of angular and size disparities were additive (see also Sekuler \& Nash, 1972).

The transformational account is limited in generality (cf., e.g., Besner, 1983; Bundesen \& Larsen, 1975; Santee \& Egeth, 1980; Simion, Bagnara, Roncato, \& Umiltà, 1982). Procedures for comparing patterns without using and transforming mental images are available, and, depending upon the choice of stimuli, some such procedure may be the most effective. Thus, to demonstrate a pure

I wish to thank my colleague Claus Bundesen for extensive comments on earlier drafts of this paper. Requests for reprints should be sent to Axel Larsen, Institute of Clinical Psychology, Copenhagen University, Njalsgade 90, DK-2300 Copenhagen S, Denmark. case of mental rotation, Shepard and Metzler (1971) used a stimulus set consisting of perspective drawings of random three-dimensional objects with negative pairs consisting of drawings of objects that were "isomeric," being mirror images of each other. The choice of random objects and isomeric negative pairs should prevent subjects from reaching a decision by retrieving and comparing name or conceptual codes for the objects to be matched. The choice of isomeric negative pairs should also prevent subjects from doing the task by discovering some local detail possessed by only one of the objects. With these precautions having been taken, the pattern of results reported by Shepard and Metzler (1971) appears highly robust (see Shepard \& Cooper, 1982).

Many experiments on mental size transformation have also used random shapes as stimuli and isomeric negative pairs. With one exception (Kubovy \& Podgorny, 1981), such experiments have confirmed the results reported by Bundesen and Larsen (1975, Experiment 2), positive and negative mean reaction times being increasing linear functions of the linear size ratio between stimuli with equal slopes for positive and negative reactions (Besner, 1983, Experiment 1; Howard \& Kerst, 1978; Larsen \& Bundesen, 1978, Experiment 1).

In the study of Kubovy and Podgorny (1981), subjects were required to decide whether two simultaneously presented random polygons were identical except for change in size and rotation in the frontoparallel plane. Positive mean reaction times increased approximately linearly with angular difference in orientation, but there was virtually no effect of size ratio for any level of angular difference in orientation. As pointed out by Kubovy and Podgorny, the experiment differed from other experiments that have shown an effect of size in that only two different random polygons (and their mirror inversions) were used throughout ( 820 trials per subject). To test whether familiarity was decisive in producing the results, I paralleled their 
work except that I used unfamiliar random shapes, a new pair on every trial.

\section{EXPERIMENT 1}

Speed of simultaneous matching of unfamiliar polygons with respect to shape regardless of differences in size and angular orientation was investigated under two conditions. In Condition A, members of the same negative pair were mirror images of each other. In Condition B, each pair consisted of two unrelated polygons. Thus, size ratio was well defined for negative pairs in Condition $A$ but not in Condition B.

Two steps were taken to provide for a tentative analysis of reaction time to negative pairs in Condition $\mathrm{A}$ as a function of "difference in angular orientation." First, each pattern in Condition A was shown with a pointer extending from its center of gravity, and this pointer was scaled in size and rotated along with the pattern such that, for any positive pair, the pointer indicated the transformation required to bring the two members into congruence. Second, presentations in Condition A were blocked by difference in angular orientation between patterns (for positive pairs) and pointers (for both positive and negative pairs).

\section{Method}

Subjects. Five subjects, including the author, participated. All subjects had normal or corrected-to-normal vision. Only the author had had previous experience with reaction time experiments.

Stimuli. The stimulus material consisted of pairs of random outline decagons displayed simultaneously side by side on the face of a computer-driven cathode ray tube (see Figure 1). The horizontal distance between the decagons' centers of gravity (defined as the first moment of area) was $5.0 \mathrm{~cm}$. The distances from a center to the closest vertical and horizontal screen borders were 3.5 and $5.0 \mathrm{~cm}$, respectively.

The random decagons were constructed by connecting neighboring endpoints of 10 evenly spaced imaginary concentric radii. For the first pattern in a pair, the radius lengths were chosen randomly from the interval $0-8 \mathrm{~mm}$, with the constraint that one radius length be selected from each of the intervals $0-0.8,0.8-1.6, \ldots$, and 7.2 $8.0 \mathrm{~mm}$.

For positive stimulus pairs, the second stimulus pattern was generated by a geometric multiplication of the first one with a factor of $1,2.5$, or 4 . By random choice, one of the two patterns was rotated clockwise in the plane by an angle of $0^{\circ}, 60^{\circ}$, or $120^{\circ}$, and this pattern was positioned in the right-hand field of the display tube; the other pattern was positioned in the left-hand field. In Condition $A$, the polygon in the left-hand field was shown with a thick vertical pointer that extended from the center of gravity through the top and was scaled to the size of the pattern. The pointer was $1.2 \mathrm{~cm}$ long in the smallest pattern. The polygon in the right-hand field was shown with a similar pointer scaled and rotated in the same way as the stimulus pattern.

Negative pairs in Condition A were constructed using the same procedure as for positive pairs except that an initial step was the left-right mirror-inverting of one member in each pair. Negative pairs in Condition B consisted of two unrelated polygons. By random choice, either the left or the right polygon was scaled in size such that the distance from the center of gravity to the most distant vertex was $1,2.5$, or 4 times as large as the corresponding distance in the other polygon.

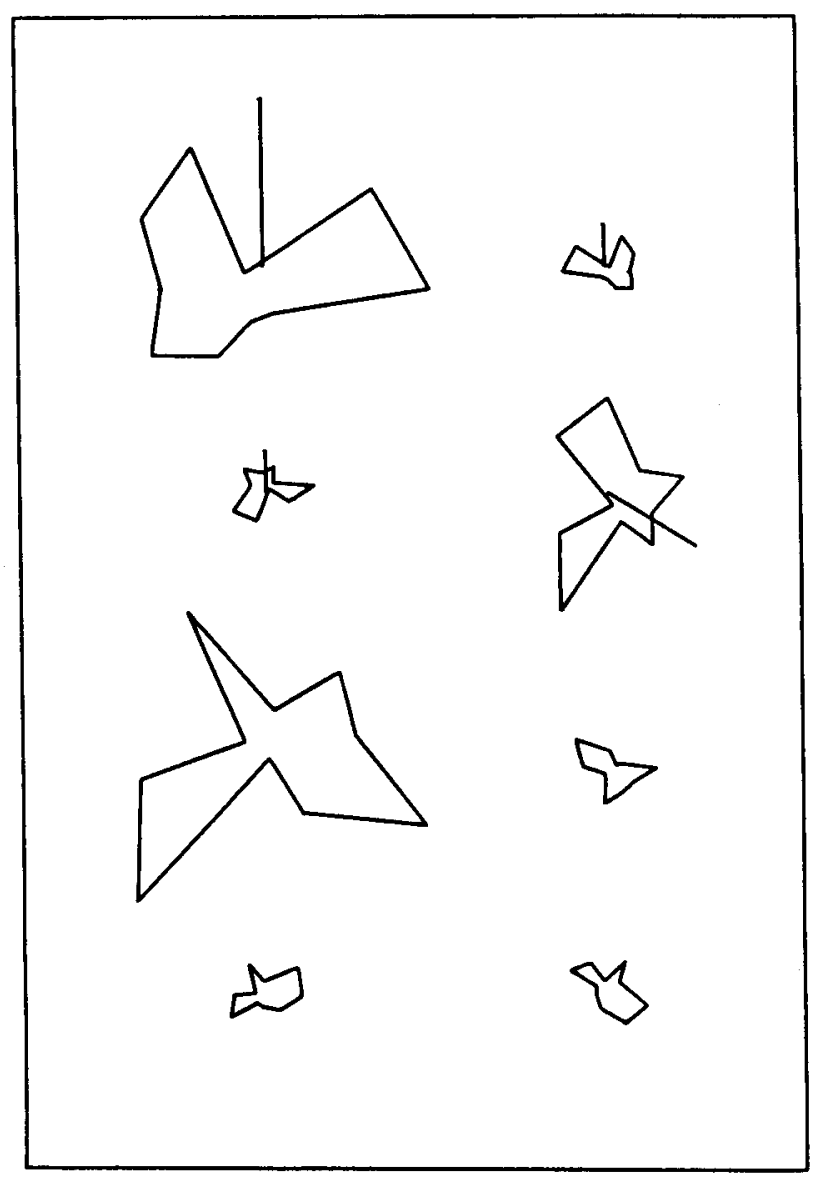

Figure 1. Examples of the stimulus material in Conditions $A$ (two upper panels) and $B$ (two lower panels).

At the chosen viewing distance of $50 \mathrm{~cm}$, a linear extent of $1 \mathrm{~cm}$ on the screen corresponds to about $1.15^{\circ}$. Thus, the smallest and the largest decagons subtended visual angles of about $1.7^{\circ}$ and $6.8^{\circ}$, respectively. The minimum horizontal distance between vertices of the two polygons was about $1.1^{\circ}$.

Design. In each experimental condition, size ratio $(1,2.5$, or 4$)$, angular difference $\left(0^{\circ}, 60^{\circ}\right.$, or $120^{\circ}$ ), and response type (positive or negative) were varied orthogonally with 72 replications of each combination of factors for each subject. For each condition and subject, the resulting 1,296 stimulus pairs were arranged in a random order of presentation with the constraint that each factor combination be repeated 12 times within each block of 216 trials. In Condition A, these blocks consisted of three subblocks of 72 stimulus pairs such that angular deviation between members of a pair was kept constant within subblocks. All randomizations were done anew for each subject, and experimental conditions were counterbalanced within subjects.

Procedure. The subjects were tested individually in two experimental sessions of up to $4 \mathrm{~h}$ each. They were instructed to strike a right-hand button " as quickly as possible"' if the displayed decagons were identical except for changes in orientation, size, or both. They were told to press a left-hand button otherwise.

The subject was seated in front of the display tube at a distance of $50 \mathrm{~cm}$ in a dimly lit room. Viewing was binocular and fixation was free. By pressing a start key, the subject initiated a block of 216 test trials preceded by 4 trials that cued the particular experimental condition. The first stimulus pair in a block was shown with 
a delay of $3 \mathrm{sec}$. Stimulus exposure terminated when a response key was pressed or after a maximum latency of $10 \mathrm{sec}$. In either case, a new pair of decagons was shown after a fixed intertrial waiting time of $1 \mathrm{sec}$. The experiment was self-paced between blocks.

\section{Results}

Analyses of reaction times were based on correct positive and negative responses in Condition $A$ and correct positive responses in Condition B. Reaction times from Condition A were subjected to a three-way (size ratio $x$ angular difference $\times$ response type) repeated measures analysis of variance. There were significant effects of size ratio $[F(2,8)=9.4, p<.01]$ and angular difference $[\mathrm{F}(2,8)=34.9, \mathrm{p}<.001]$, but not of response type $[F(1,4)=2.3, p>.10]$. No interactions were significant (ps $>.25$ ). Analysis of trends showed significant linear components of the main effects of size ratio $[F(1,8)$ $=18.5, \mathrm{p}<.005]$ and angular difference in orientation $[F(1,8)=69.3, p<.001] ;$ higher order trends did not reach significance $(\mathrm{Fs}<1)$. The results are summarized across subjects and response types in the left-hand panel of Figure 2.

Positive reaction times for Condition B were subjected to a two-way (size ratio $X$ angular difference) repeated measures analysis of variance. There were significant effects of size ratio $[F(2,8)=12.5, p<.005]$ and angular difference $[F(2,8)=21.6, p<.001]$, but the interaction was not significant $(F<1)$. Linear components of the effects of size ratio and angular difference were significant $[F(1,8)=24.9, p=.005$, and $F(1,8)=42.9$, $p<.001$, respectively], but higher order trends were not (Fs $<1)$. The results are summarized across subjects in the right-hand panel of Figure 2.

In either experimental condition, many reaction times were unusually long. However, the reported data appear robust. Introducing a cutoff limit for outliers at $4,000 \mathrm{msec}$

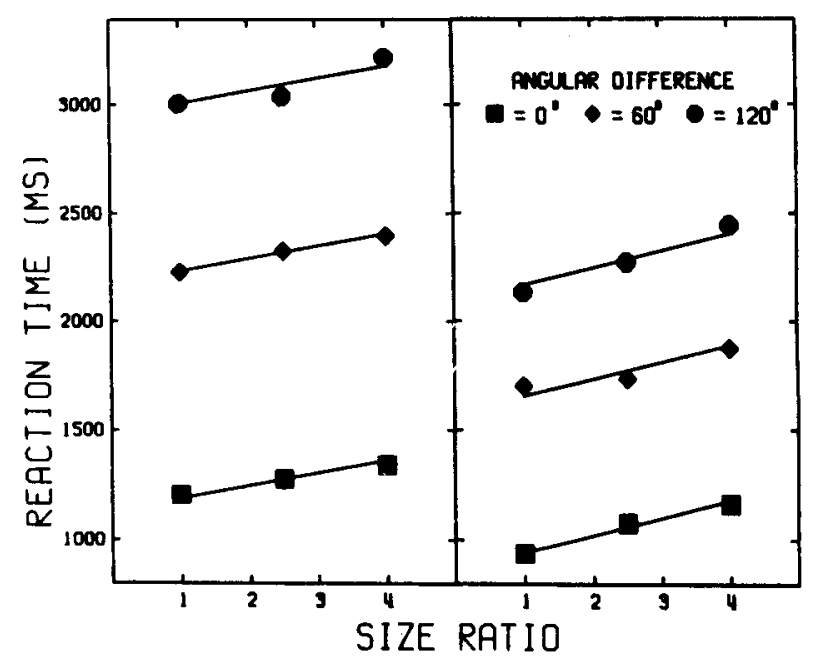

Figure 2. Mean reaction times as functions of size ratio with angular difference in orientation as parameter. Left panel: Results from Condition $A$ across response types (positive vs. negative). Right panel: Results from Condition B for positive responses. In both panels, data are fitted by parallel straight lines.
Table 1

Mean Percentage of Errors as Functions of Size Ratio and Angular Difference in Orientation for Conditions $A$ and $B$ in Experiment 1

\begin{tabular}{lcrrr}
\hline & Angular & \multicolumn{3}{c}{ Size Ratio } \\
& Difference & 1.0 & 2.5 & 4.0 \\
\hline \multirow{4}{*}{ False negatives } & \multicolumn{2}{c}{ Condition A } & & \\
& $0^{\circ}$ & 3.9 & 2.8 & 2.8 \\
& $60^{\circ}$ & 4.2 & 4.4 & 6.4 \\
False positives & $120^{\circ}$ & 11.7 & 13.9 & 12.0 \\
& $0^{\circ}$ & 9.4 & 5.6 & 5.1 \\
& $60^{\circ}$ & 16.7 & 11.4 & 14.2 \\
& $120^{\circ}$ & 20.9 & 18.6 & 19.2 \\
False negatives & Condition B & & \\
& $0^{\circ}$ & 0.3 & 1.4 & 1.9 \\
& $60^{\circ}$ & 3.3 & 6.7 & 6.9 \\
False positives & $120^{\circ}$ & 12.2 & 15.8 & 18.6 \\
& - & 5.0 & 7.2 & 5.4 \\
\hline
\end{tabular}

Note-The rate of false-positive responses in Condition $B$ averaged $5.9 \%$. In this condition, neither size ratio nor angular difference were defined.

would lead to rejection of a total of 930 reaction times (7.2\%), but the statistical conclusions would not be affected. Also, the pattern of medians was highly similar to that of the means.

Error rates for Conditions $\mathrm{A}$ and $\mathrm{B}$ are given in Table 1 . Both positive and negative error rates showed strong increase with angular difference in orientation $\left[\chi^{2}(10)=106, \mathrm{p}<.001\right.$, for false negative responses in Condition $A ; \chi^{2}(10)=100, p<.001$, for false positive responses in Condition $A ; \chi^{2}(10)=188, p<.001$, for false negatives in Condition $B]$. Effects of size ratio were relatively weak $\left[\chi^{2}(10)=11, p=.36\right.$, for false negatives in Condition $A ; \chi^{2}(10)=15.6, p=.11$, for false positives in Condition $A ; \chi^{2}(10)=21.8, p=.02$, for false negatives in Condition $\mathbf{B}$ ]

\section{Discussion}

The main results are clear: Mean latencies for simultaneous matching of unfamiliar random polygons with respect to shape showed linear and additive effects of size ratio and angular difference in orientation within stimulus pairs. With a new pair of random polygons presented on every trial, the effects were seen in positive reaction times whether members of negative pairs were mirror images of each other (Condition A) or unrelated polygons (Condition B). When bar pointers indicated the transformation required to bring members of a positive pair into congruence (or sufficient to establish incongruence between members of negative pairs) and presentations were blocked by difference in angular orientation (Condition A), the effects seen in positive reaction times were also apparent in negative reaction times.

The pattern of simultaneous matching reaction times found for unfamiliar random polygons in Experiment 1 is the same as that obtained by Bundesen et al. (1981) for successive matching of alphanumeric characters with mirror-image negative pairs and bar pointers indicating the transformation required to bring positive pairs into congruence. However, mean reaction times in the present 
experiment (ranging from about $1 \mathrm{sec}$ to about $3.5 \mathrm{sec}$ ) were much longer than mean reaction times in the previous experiment (ranging from about $450 \mathrm{msec}$ to about $900 \mathrm{msec}$ ). Considerably faster successive than simultaneous matching reaction times have been found in many other studies (compare, e.g., the successive matching results of Larsen \& Bundesen, 1978, Experiment 1, against the simultaneous matching results of Bundesen \& Larsen, 1975, Experiment 2, for essentially the same stimulus patterns). It appears that the time taken to complete a mental-image transformation procedure for comparing two patterns is greatly reduced once a good mental image of one of the patterns has been established.

The results of Experiment 1 contrast with those of Kubovy and Podgorny (1981). I conjecture that the difference in familiarity between stimulus materials in the two experiments was crucial. Apparently, for unfamiliar patterns (Experiment 1), a simple mental-image transformation procedure was the most efficient. For familiar patterns (Kubovy \& Podgorny, 1981), decision procedures based on matching against representations in long-term memory (cf., e.g., Larsen \& Bundesen, 1978) or specialized diagnostic strategies (cf., e.g., Fox, 1975) may be more efficient than the simple mental-image transformation procedure. Note that, given this account, the results of Kubovy and Podgorny suggest that the pattern of data obtained by Bundesen et al. (1981) for (familiar) alphanumeric characters in successive matching conditions (in which mental-image transformation procedures are relatively fast) would break down in simultaneous matching conditions (in which image transformation procedures are relatively slow).

Experiment 2 was designed to further test the conjecture that familiarity was decisive in producing the Kubovy and Podgorny (1981) pattern of results. I hoped to be able to reproduce the contrasting results of Experiment 1 and the experiment by Kubovy and Podgorny within a single experimental setting.

\section{EXPERIMENT 2}

Two experimental conditions for simultaneous matching of random polygons with respect to shape regardless of size and orientation were contrasted. In both conditions, members of the same negative pair were mirror images of each other. Throughout Condition A, each subject worked with only two different polygons and their mirror inversions. In Condition B, a new pair of unfamiliar random polygons was presented on every trial. The two conditions were the same in all other respects.

\section{Method}

The method was identical to that used in the previous experiment except as indicated below.

Subjects. Three of the subjects from Experiment 1, including the author, and six new subjects with normal or corrected-to-normal vision participated. Two of the new subjects had had previous experience with reaction time experiments.
Stimuli. As in Experiment 1, random outline decagons were constructed by connecting neighboring endpoints of 10 evenly spaced imaginary concentric radii ranging between 0 and $8 \mathrm{~mm}$ in length. A positive pair was generated by copying the same decagon into the two fields of the display tube, scaling each of the two patterns independently by a factor of 1,2 , or 4 , and rotating the right-hand pattern clockwise by an angle of $0^{\circ}, 60^{\circ}$, or $120^{\circ}$. A negative pair was constructed using the same procedure except that an initial step was the left-right mirror-inverting of one of the decagons in a pair.

Design. Throughout Condition A, each subject worked with only two random decagons and their left-right mirror inversions (different decagons for different subjects). Following Kubovy and Podgorny (1981), each display had one of these four shapes in the left-hand field at one of two orientations $\left(0^{\circ}\right.$ or $\left.180^{\circ}\right)$ such that the eight versions (four shapes $\times$ two orientations) were equally frequent. Departing from Kubovy and Podgorny, the size of the pattern in the left-hand field was varied. In Condition B, every trial presented a new polygon. Otherwise, the two conditions were identical.

In each experimental condition, size of left-hand pattern $(1,2$, or 4$)$, size of right-hand pattern $(1,2$, or 4$)$, angular difference in orientation $\left(0^{\circ}, 60^{\circ}\right.$, or $\left.120^{\circ}\right)$, and response type (positive vs. negative) were varied orthogonally with 24 replications for each combination of factors for each subject. For each condition and subject, the resulting 1,296 stimulus pairs were arranged in a random order of presentation divided into two blocks of 648 trials with the constraint that each factor combination be repeated once within each subblock of 54 trials. Ordering of the four blocks was counterbalanced (ABBA or BAAB) within subjects with respect to condition. Two successive blocks ( $\mathrm{AB}$ or $\mathrm{BA}$ ) formed one experimental session.

\section{Results}

Mean reaction time for correct positive responses in Condition A is shown in the left-hand panel of Figure 3 as a function of size ratio with angular difference in orientation within stimulus pairs as a parameter. For pairs of stimuli in the same orientation, reaction time increased with size ratio, but for pairs with angular differences in orientation of $60^{\circ}$ or $120^{\circ}$, effects of size ratio were

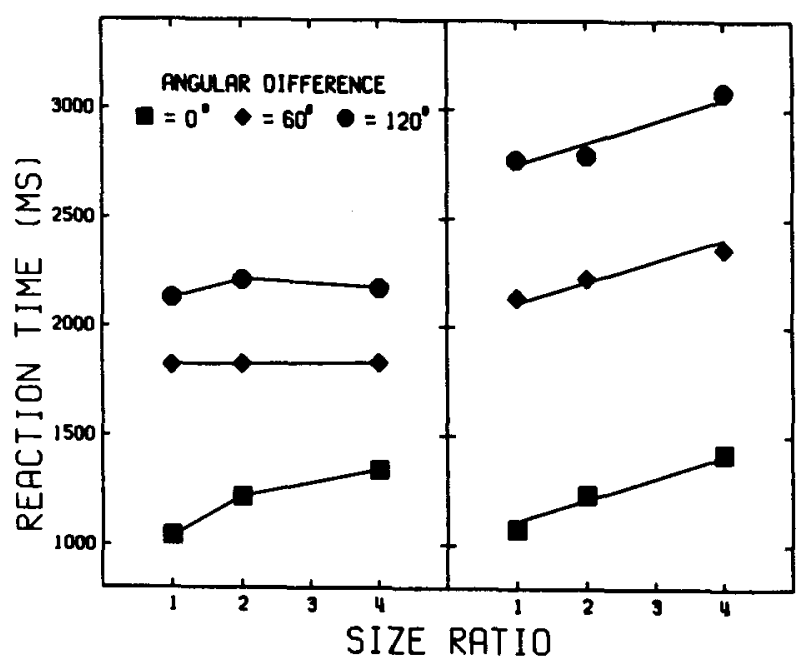

Figure 3. Mean positive reaction times as functions of size ratio with angular difference in orientation as parameter. Left panel: Results from Condition A. Right panel: Results from Condition B fitted by parallel straight lines. 
negligible. For all levels of size ratio, reaction time increased with angular difference.

By a two-way (size ratio $x$ angular difference) repeated measures analysis of variance, the main effect of size ratio $[F(2,16)=3.8, p<.05]$, the main effect of angular difference $[F(2,16)=27.7, p<.001]$, and their interaction $[F(4,32)=4.2, p<.01]$ were significant. By separate analyses, the effect of size ratio was significant for pairs of stimuli in the same orientation $[F(2 ; 16)$ $=13.1, \mathrm{p}<.001]$, but not for other pairs $(\mathrm{F}<1)$.

Mean reaction time for correct positive responses in Condition B is shown in the right-hand panel of Figure 3 as a function of size ratio with angular difference as a parameter. As in Experiment 1, effects of size ratio and angular difference were approximately linear and additive.

A two-way (size ratio $x$ angular difference) repeated measures analysis of variance established the significance of the effects of size ratio $[\mathrm{F}(2,16)=19.2, \mathrm{p}<.001]$ and angular difference $[F(2,16)=74.3, p<.001]$ and lack of interaction $(\mathrm{F}<1)$. Analyses of trends showed reliability of the linear components of the effects of size ratio $[F(1,16)=38.3, p<.001]$ and angular difference $[F(1,16)=146.3, p<.001]$. Quadratic trends did not reach significance [for size ratio, $\mathrm{F}<1$; for angular difference, $\mathrm{F}(1,16)=2.35, \mathrm{p}>.10]$.

Mean reaction times for correct positive responses were contrasted between Conditions A and B in a three-way (condition $\times$ size ratio $X$ angular difference) repeated measures analysis of variance. This analysis showed that the two conditions differed with respect to grand mean (smaller in Condition A), effect of size ratio (weaker in Condition A), and effect of angular difference (weaker in Condition A). Thus, there were significant effects of condition $[F(1,8)=14.4, p<.01]$, size ratio $[F(2,16)$ $=16.4, \mathrm{p}<.001]$, and angular difference $[\mathrm{F}(2,16)=$ $57.2, \mathrm{p}<.001]$, and significant interactions between condition and size ratio $[F(2,16)=5.6, p<0.5]$ and between condition and angular difference $[\mathrm{F}(2,16)=20.6$, $\mathrm{p}<.001]$. The interaction between size ratio and angular difference did not reach significance $[F(4,32)=2.56$, $p>.05$ ], nor did the three-way interaction between condition, size ratio, and angular difference $[F(4,32)=1.82$, $\mathrm{p}>.10]$.

Mean reaction times for correct negative responses were longer than mean reaction times for positive ones, but product-moment correlations between positive and negative response latencies across the factorial combinations of size ratio and difference in angular orientation were high. In Condition A, positive and negative grand means were 1,735 and $2,143 \mathrm{msec}$ and the correlation was .96. In Condition B, the grand means were 2,105 and $2,609 \mathrm{msec}$ and the correlation was .93 .

Error rates for Conditions A and B are shown in Table 2. Both positive and negative error rates increased with angular difference in orientation, particularly in Condition $B\left[\chi^{2}(18)=145, p<.001\right.$, for false negative responses in Condition $A ; \chi^{2}(18)=58, p<.001$, for false positive responses in Condition $A ; \chi^{2}(18)=195$,
Table 2

Mean Percentage of Errors as a Function of Size Ratio and Angular Difference in Orientation for Conditions $A$ and $B$ in Experiment 2

\begin{tabular}{lcrrr}
\hline & Angular & \multicolumn{3}{c}{ Size Ratio } \\
& Difference & 1 & 2 & 4 \\
\hline \multirow{4}{*}{ False negatives } & Condition A & & \\
& $0^{\circ}$ & 1.9 & 1.7 & 4.2 \\
& $60^{\circ}$ & 6.4 & 6.6 & 6.3 \\
False positives & $120^{\circ}$ & 4.2 & 4.4 & 5.4 \\
& $0^{\circ}$ & 0.5 & 1.6 & 2.1 \\
& $60^{\circ}$ & 7.3 & 5.8 & 4.4 \\
& $120^{\circ}$ & 7.1 & 4.6 & 7.4 \\
False negatives & Condition B & & \\
& $0^{\circ}$ & 4.6 & 5.4 & 6.0 \\
& $60^{\circ}$ & 11.1 & 10.5 & 13.0 \\
False positives & $120^{\circ}$ & 16.4 & 15.6 & 18.6 \\
& $0^{\circ}$ & 1.5 & 1.5 & 1.2 \\
& $60^{\circ}$ & 7.6 & 5.6 & 6.3 \\
& $120^{\circ}$ & 11.3 & 12.0 & 10.2 \\
\hline
\end{tabular}

$\mathrm{p}<.001$, for false negatives in Condition $\mathrm{B} ; \chi^{2}(18)$ $=165, \mathrm{p}<.001$, for false positives in Condition B]. The rate of false negatives increased as a function of size ratio in Condition $\mathrm{A}$; otherwise effects of size ratio were weak $\left[\chi^{2}(18)=36, p<.01\right.$, for false negatives in Condition $\mathrm{A} ; \chi^{2}(18)=17.1, \mathrm{p}=.52$, for false positives in Condition $\mathrm{A} ; \chi^{2}(18)=13.7, \mathrm{p}=.75$, for false negatives in Condition $B ; \chi^{2}(18)=19.6, p=.36$, for false positives in Condition B].

\section{Discussion}

Conditions A and B differed in one and only one respect: Throughout Condition A, each subject worked with only two different random shapes and their mirror inversions; in Condition B, a new pair of unfamiliar random shapes was presented on every trial. This difference caused a clear difference in performance. Matching reaction times in Condition B showed strong linear and additive effects of size ratio and angular difference in orientation within stimulus pairs. Matching reaction times in Condition A were faster, effects of size ratio and angular difference were much weaker, and the two effects appeared to interact such that for angular differences in orientation of $60^{\circ}$ or $120^{\circ}$, effects of size ratio were negligible.

I had expected to be able to replicate the contrasting results of Experiment 1 and the study of Kubovy and Podgorny (1981) within Experiment 2. The results from Condition B did replicate the pattern of matching latencies seen in Experiment 1, suggesting that, for unfamiliar shapes, a simple mental-image transformation procedure was the most efficient. The results from Condition A replicated the pattern of matching reaction times reported by Kubovy and Podgorny (1981) for angular differences of $60^{\circ}$ and $120^{\circ}$ but not for pairs of stimuli in the same angular orientation. Although the replication is less than perfect, the contrast between Conditions A and B (the effect of using the same few random shapes again and again) 
strongly suggests that familiarity was of major importance in producing the Kubovy and Podgorny pattern of results.

The data of Kubovy and Podgorny (1981) suggested that their subjects used a procedure based on mental rotation but not on mental size transformation. A possible procedure of this sort is as follows: Given two patterns to be compared, locate three (noncollinear) characteristic points ( $A, B$, and $C$ ) in one of the patterns and the corresponding points $\left(A^{\prime}, B^{\prime}\right.$, and $\left.C^{\prime}\right)$ in the other pattern. Rotate the first pattern in the plane such that $A$ is mapped onto $A^{\prime}$ and line segment $\mathbf{A B}$ gets aligned with $A^{\prime} B^{\prime}$. If, and only if, the rotation brings segment $A C$ into alignment with segment $\mathrm{A}^{\prime} \mathrm{C}^{\prime}$, then the two patterns form a positive pair. Note two points: First, a size transformation is not required to judge whether two segments are aligned. Second, the complexity of the procedure is embedded in the first step, finding corresponding points in the two patterns. Recognition of corresponding landmarks in the two patterns should be greatly facilitated by familiarity of these patterns.

\section{GENERAL DISCUSSION}

Consider the evidence on mental size transformation from matching reaction-time studies using random shapes as stimuli and isomeric negative pairs. When precautions have been taken to minimize effects of familiarity by using large numbers of different random shapes, all such experiments have yielded the same pattern of results. To a good approximation, positive and negative mean reaction times have been linearly increasing functions of the linear size ratio within stimulus pairs and the two functions have had the same slope (Besner, 1983, Experiment 1; Bundesen \& Larsen, 1975, Experiment 2; Howard \& Kerst, 1978; Larsen \& Bundesen, 1978, Experiment 1). This pattern of results strongly suggests that the matching task in question is performed by mentally transforming one member of a stimulus pair into the size format of the other one and then testing for a match.

Besner and Coltheart (1976) attempted an alternative explanation for the results of Bundesen and Larsen (1975, Experiment 2). The Besner and Coltheart model assumes that subjects initially make a global estimate of the general similarity between the stimulus patterns to be compared. To explain increase in positive reaction time as a function of size ratio, it is hypothesized that this similarity estimate serves to raise the decision criteria associated with the positive response such that similarity reduces this threshold, whereas evidence of a difference raises it. It is assumed that size disparity thus biases subjects against positive responses. With this assumption, one might also expect that size disparity biases subjects towards negative responses, which contradicts data that shows increasing negative reaction times as a function of size ratio.

To accommodate these data, Besner and Coltheart (1976) suggested that such negative responses were con- trolled by a clock reaching some deadline $t$, where $t$ is preset according to the initial estimate of similarity: The greater the dissimilarity, the more time is set to pass before a negative response is evoked. Besides being ad hoc, the model implies that, for a given level of size disparity (e.g., same-sized pairs), negative reaction time should increase with increasing dissimilarity between the patterns to be compared, which is hardly true.

Besner and Coltheart (1976) concluded that "it might be premature to assume that Bundesen and Larsen's (1975) results unequivocally support a normalization hypothesis to the exclusion of all other explanations" (p. 530). I agree with this conclusion. On the other hand, the alternative model proposed by Besner and Coltheart appears deficient, and I am not aware of any other serious attempts to account for the pattern of matching reaction times obtained with random shapes as stimuli and isomeric negative pairs when precautions are taken to eliminate effects of familiarity.

\section{REFERENCES}

BESNER, D. (1983). Visual pattern recognition: Size prepocessing reexamined. Quarterly Journal of Experimental Psychology, 35A, 209-216.

Besner, D., \& Coltheart, M. (1976). Mental size scaling examined. Memory \& Cognition, 4, 525-531.

BUNDESEN, C., \& LARSEN, A. (1975). Visual transformation of size. Journal of Experimental Psychology: Human Perception \& Performance, 1, 214-220.

Bundesen, C., Larsen, A., \& Farrell, J. E. (1981). Mental transformations of size and orientation. In J. Long \& A. Baddeley (Eds.), Attention and performance (Vol. 9, pp. 279-294). Hillsdale, NJ: Erlbaum.

Fox, J. (1975). The use of structural diagnostics in recognition. Journal of Experimental Psychology: Human Perception \& Performance, 1, 57-67.

Howard, J. H., \& Kerst, S. M. (1978). Directional effects of size changes on the comparison of visual shapes. American Joumal of Psychology, 91, 491-499.

Kubovy, M., \&odgorny, P. (1981). Does pattern matching require the normalization of size and orientation? Perception \& Psychophysics, 30, 24-28.

LARSEN, A., \& Bundesen, C. (1978). Size scaling in visual pattern recognition. Joumal of Experimental Psychology: Human Perception \& Performance, 4, 1-20.

SANTEE, J. L., \& EGETH, H. E. (1980). Selective attention in the speeded classification and comparison of multidimensional stimuli. Perception \& Psychophysics, 28, 191-204.

SEKUlER, R., \& NASH, D. (1972). Speed of size scaling in human vision. Psychonomic Science, 27, 93-94.

Shepard, R. N., \& COOPER, L. A. (1982). Mental images and their transformations. Cambridge, MA: MIT Press/Bradford Books.

ShePARD, R. N., \& Metzler, J. (1971). Mental rotation of threedimensional objects. Science, 171, 701-703.

Simion, F., Bagnara, S., Roncato, S., \& Umiltà, C. (1982). Transformation processes upon the visual code. Perception \& Psychophysics, $31,13-25$.

(Manuscript received January 29, 1985; revision accepted for publication June 24,1985 .) 